\title{
On the degree of geodesic mobility of Riemannian manifolds
}

\author{
Elena Stepanova and Marie Chodorová
}




\title{
ON THE DEGREE OF GEODESIC MOBILITY OF RIEMANNIAN MANIFOLDS
}

\author{
ELENA STEPANOVA AND MARIE CHODOROVÁ
}

\begin{abstract}
We will consider the definition of the degree $r$ of geodesic mobility of a Riemannian manifold and will prove that the well-known results on the geodesic mobility are essentially local. In the last section of our paper, we will prove that the geodesic mobility of an $n$-dimensional closed, simply-connected Riemannian manifold with positive constant sectional curvature satisfies the identity $r=(n+1)(n+2) / 2, n \geq 2$.
\end{abstract}

2000 Mathematics Subject Classification: 53C20; 53C21; 53C22

Keywords: Riemannian manifold, geodesic mapping, degree of geodesic mobility

\section{INTRODUCTION}

The solving of the problem of geodesic mapping of Riemannian manifolds was started by Levi-Civita (see [5]) who studied the trajectory equivalence of dynamical systems. The definition of the degree of geodesic mobility of a Riemannian manifold was introduced by Sinyukov (see [12, p. 137], see [2,8], [11, p. 168]) as a numerical characteristic of the cardinality of the geodesic class. The method for finding the degree of geodesic mobility is based on an analysis of the integrability conditions of fundamental equations of geodesic mappings (see [12, pp. 137-147]). Interesting results of global geodesic mappings for special Riemannian manifolds are formulated in papers $[1,6-11]$.

In this paper, we will prove that the well-known results on the geodesic mobility are essentially local and that the geodesic mobility $r$ of an $n$-dimensional closed, simply-connected Riemannian manifold with positive constant sectional curvature satisfies the identity

$$
r=(n+1)(n+2) / 2 .
$$

\section{THE DEGREE OF GEODESIC MOBILITY}

Suppose that $(M, g)$ is an $n$-dimensional Riemannian manifold and $\nabla$ is the LeviCivita connection corresponding to the metric tensor $g=\left(g_{i j}\right), n \geq 2$.

The paper was supported by Grant PrF-2013-033 (Mathematical structures) and by the project POST-UP CZ 1.07/2.3.00/30.0004. 
A mapping of the Riemannian manifold $(M, g)$ to a Riemannian manifold $\left(M^{\prime}, g^{\prime}\right)$ is called geodesic if it takes every geodesic of $(M, g)$ to a geodesic of $\left(M^{\prime}, g^{\prime}\right)$.

It is well known (see, for example [12, p. 137], [8], [11, p. 168]) that the Riemannian manifold $(M, g)$ admits a geodesic mapping if and only if there exists a covariant regular symmetric tensor field $a=\left(a_{i j}\right)$ on this manifold such that

$$
\nabla_{k} a_{i j}=\lambda_{i} g_{j k}+\lambda_{j} g_{i k}
$$

where $\lambda_{i}=\frac{1}{2} \nabla_{i}\left(\operatorname{trace}_{g} a\right)$. If $\lambda_{i}=0$, then the geodesic mapping is called trivial.

The equations (2.1) have been completed by Sinyukov to a system of differential equations of Cauchy type in covariant derivatives (see [12, p. 134]; [8]; [11, p. 168]). In particular, Sinyukov has proved that if the system of differential equations (2.1) is a totally-integrable system of differential equations in a neighborhood of an arbitrary point of Riemannian manifold $(M, g)$, then $(M, g)$ is a manifold of constant curvature (see [12, p. 138]).

The degree of geodesic mobility $r$ of an $n$-dimensional Riemannian manifold $(M, g)$ is the dimension of linear space of solutions of system of differential equations (2.1). The notion of geodesic mobility of a Riemannian manifold was introduced by Sinyukov (see [12, p. 137], [8]). From Sinyukov's theorem, we conclude that the $n$ dimensional Riemannian manifolds of constant curvature and only these manifolds have the maximal degree $r=(n+1)(n+2) / 2$ for $n \geq 2$.

On the other hand, an arbitrary Riemannian manifold has mobility no smaller than 1 , because system (2.1) has at least one solution of the form $a=\alpha g$ for an arbitrary real constant $\alpha$ on this manifold. Therefore, we can conclude that $1 \leq r \leq(n+$ 1) $(n+2) / 2$ for any $n$-dimensional Riemannian manifold $(M, g)$.

The result formulated in Sinyukov's theorem and other results on the geodesic mobility (see [11, pp. 147-149]) are essentially local. For example, we consider an $n$-dimensional hyperbolic space, which is a Riemannian manifold $(M, g)$ of constant negative curvature. In this case, we have $r=(n+1)(n+2) / 2$. If we factorize hyperbolic space by a suitable discrete group of motions (see [13]), we obtain a compact manifold of constant negative curvature.

It is well known (see [7]) that on a compact Riemannian manifold with non positive constant curvature, any tensor field $a=\left(a_{i j}\right)$ satisfying the equations (2.1) has the trivial form $a=$ const $\cdot g$. Therefore, this manifold does not admit a "globally defined" non-trivial geodesic mapping, i. e. $r=1$. Finally, we can conclude that the number $r=(n+1)(n+2) / 2$ is a local numerical characteristic of the cardinality of the geodesic class of a hyperbolic space. On the other hand, if we consider the number $r$ as a global numerical characteristic of the cardinality of the geodesic class of a compact hyperbolic space, then $r=1$.

\section{THE MAIN THEOREM}

In this section, we shall prove the main proposition of our paper. 
Theorem. Let $(M, g)$ be an $n$-dimensional closed and simply-connected Riemannian manifold with positive constant sectional curvature and $r$ be the degree of geodesic mobility as a global numerical characteristic of the cardinality of the geodesic class of $(M, g)$. Then

$$
r=(n+1)(n+2) / 2 .
$$

Proof. Let $\mathbb{R}^{n+1}$ be an $(n+1)$-dimensional Euclidean space endowed with the inner product $\langle\cdot, \cdot\rangle$. If $\left\{x^{\alpha}\right\}$ for $\alpha, \beta, \gamma=1, \ldots, n, n+1$ are Cartesian orthogonal coordinates in $\mathbb{R}^{n+1}$ then

$$
\mathbb{S}^{n}: \quad\left(x^{1}\right)^{2}+\cdots+\left(x^{n}\right)^{2}+\left(x^{n+1}\right)^{2}=1
$$

is a unit sphere in $\mathbb{R}^{n+1}$. If the sphere $\mathbb{S}^{n}$ is represented locally by $x^{\alpha}=f^{\alpha}\left(u^{k}\right)$ in terms of its local coordinates $\left\{u^{k}\right\}$, then putting $f_{k}^{\alpha}=\partial_{k} x^{\alpha}$ for $\partial_{k}=\partial / \partial u^{k}$, we can define the second fundamental form of the sphere $\mathbb{S}^{n}$ by the Gauss equations (see [14])

$$
f_{i j}^{\alpha}=\nabla_{i} f_{j}^{\alpha}=\partial_{i} f_{j}^{\alpha}-f_{k}^{\alpha} \Gamma_{j i}^{k}+\Gamma_{\beta \gamma}^{\alpha} f_{i}^{\beta} f_{j}^{\gamma}
$$

where $\nabla_{i}$ is the van der Waerden-Bortolotti covariant differentiation along $\mathbb{S}^{n}, \Gamma_{j i}^{k}$ are the Christoffel symbols of the metric tensor $g_{i j}=f_{i}^{\alpha} f_{j}^{\beta} g_{\alpha \beta}$ on $\mathbb{S}^{n}, \Gamma_{\beta \gamma}^{\alpha}$ are the Christoffel symbols of the metric tensor $g_{\alpha \beta}$ on a $(n+1)$-dimensional Euclidean space. In a Cartesian orthogonal system, $g_{\alpha \beta}=\delta_{\alpha \beta}$ and $\Gamma_{\beta \gamma}^{\alpha}=0$, where $\delta_{\alpha \beta}$ is Kronecker's delta.

It is well known that $\mathbb{S}^{n}$ is a totally umbilical submanifold and hence $f_{i j}^{\alpha}=g_{i j} N^{\alpha}$, where $N^{\alpha}=-x^{\alpha}$ are the components of the unit normal vector $N \in C^{\infty}\left(T \Phi^{n}\right)^{\perp}$.

If $A=\left(A_{\alpha \beta}\right)$ is a constant symmetric tensor, then we can define a tensor field $a=\left(a_{i j}\right)$ on $\mathbb{S}^{n}$ by the following identities $a_{i j}=A_{\alpha \beta} f_{i}^{\alpha} f_{j}^{\beta}$. Applying $\nabla_{k}$ to these identities, we obtain

$$
\begin{aligned}
\nabla_{k} a_{i j} & =f_{k}^{\gamma}\left(\nabla_{\gamma} A_{\alpha \beta}\right) f_{i}^{\alpha} f_{j}^{\beta}+A_{\alpha \beta}\left(f_{k i}^{\alpha} f_{j}^{\beta}+f_{i}^{\alpha} f_{k j}^{\beta}\right) \\
& =A_{\alpha \beta}\left(N^{\alpha} f_{j}^{\beta} g_{i k}+N^{\beta} f_{i}^{\alpha} g_{j k}\right) .
\end{aligned}
$$

If we suppose that $\lambda_{j}=A_{\alpha \beta} N^{\alpha} f_{j}^{\beta}$, then we can rewrite the last equations in the following form

$$
\nabla_{k} a_{i j}=\lambda_{i} g_{j k}+\lambda_{j} g_{i k}
$$

which shows that $\mathbb{S}^{n}$ admits a globally defined geodesic mapping because $a=\left(a_{i j}\right)$ is a tensor field globally defined on $\mathbb{S}^{n}$.

The number of independent components of the constant symmetric tensor $A=$ $\left(A_{\alpha \beta}\right)$ is equal to $(n+1)(n+2) / 2$. Therefore, we can determine a sharp upper bound on the degree of geodesic mobility $r$ as a global numerical characteristic of the cardinality of the geodesic class for a unit sphere $\mathbb{S}^{n}$. In addition, we recall that Hopf has shown (see $[3,4]$ ) that a closed, simply-connected Riemannian manifold 
with constant sectional curvature 1 is necessarily isometric to the unit sphere $\mathbb{S}^{n}$, equipped with its standard metric. This finishes the proof.

\section{REFERENCES}

[1] I. Hinterleitner, "On global geodesic mappings of ellipsoids," AIP Conf. Proc., vol. 1460, pp. 180-184, 2012.

[2] I. Hinterleitner and J. Mikeš, "Fundamental equations of geodesic mappings and their generalizations," J. Math. Sci., vol. 174, pp. 537-554, 2011.

[3] H. Hopf, "Zum Clifford-Kleinschen Raumproblem," Math. Ann., vol. 95, pp. 313-339, 1926.

[4] H. Hopf, "Differentialgeometrie und topologische Gestalt," Jahresber. Deutsch. Math.-Verein., vol. 41, pp. 209-229, 1932.

[5] T. Levi-Civita, "Sulle transformatzioni delle equazioni dinamiche," Annali Mat., vol. 60, no. 2, pp. 255-300, 1896.

[6] J. Mikeš, "On the existence of n-dimensional compact Riemannian spaces admitting nontrivial global projective transformations," Sov. Math. Dokl., vol. 39, no. 2, pp. 315-317, 1989.

[7] J. Mikeš, "Global geodesic mappings and their generalizations for compact Riemannian spaces," in Differential Geometry and its Applications, Proc. Conf., Opava, Czechoslovakia, Aug. 1992. Opava: Silesian Univ. Math. Publ., 1993, pp. 143-149.

[8] J. Mikeš, "Geodesic mappings of affine-connected and Riemannian spaces," J. Math. Sci., vol. 78, no. 3, pp. 311-333, 1996.

[9] J. Mikeš and M. Chodorová, "On concircular and torse-forming vector fields on compact manifolds,” Acta Math. Acad. Paedagog. Nyházi. (N.S.), vol. 26, no. 2, pp. 329-335, 2010.

[10] J. Mikeš and K. Strambach, "Differentiable structures on elementary geometries," Result. Math., vol. 53, no. 1-2, pp. 153-172, 2009.

[11] J. Mikeš, A. Vanžurová, and I. Hinterleitner, Geodesic mappings and some generalizations. Olomouc: Palacky University Press, 2009.

[12] N. S. Sinyukov, Geodesic mappings of Riemannian spaces. Moscow: Nauka, 1979.

[13] J. A. Wolf, Space of constant curvature. Berkley: Univ. of California, 1972.

[14] K. Yano, "Submanifolds with parallel mean curvature vector of a Euclidean space or a sphere," Kodai Math. Sem. Rep., vol. 23, pp. 144-159, 1971.

\section{Authors' addresses}

Elena Stepanova

Palacky University, Department of Algebra and Geometry, 17. listopadu 12, 77146 Olomouc, Czech Republic

E-mail address: stephelena@list.ru

Marie Chodorová

Palacky University, Department of Algebra and Geometry, 17. listopadu 12, 77146 Olomouc, Czech Republic

E-mail address: marie.chodorova@upol.cz 